\title{
Case Report: Photobiomodulation With Customized Insoles on Maximum Plantar Pressure in Diabetic Foot Syndrome by the Biomechanical Model: A Case Report
}

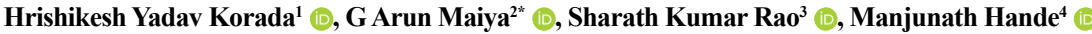

1. Centre for Diabetic Foot Care and Research, Department of Physiotherapy, Manipal College of Health Professions, Manipal Academy of Higher Education, Manipal, Karnataka, India.

2. Department of Physiotherapy, Manipal College of Health Professions, Centre for Diabetic Foot Care and Research, Kasturba Hospital, Manipal Academy of Higher Education, Manipal, Karnataka, India.

3. Department of Orthopedics, Kasturba Medical College, Manipal Academy of Higher Education, Manipal, Karnataka, India.

4. Comprehensive Geriatric Clinic, Kasturba Medical College, Manipal Academy of Higher Education, Manipal, Karnataka, India.

\begin{tabular}{|c|c|}
\hline $\begin{array}{l}\text { Use your device to scan } \\
\text { and read the article online }\end{array}$ & Citation: Yadav Korada H, Maiya GA, Kumar Rao S, Hande M. Photobiomodulation With Customized Insoles on Maximum \\
\hline 回的部回 & $\begin{array}{l}\text { Plantar Pressure in Diabetic Foot Syndrome by the Biomechanical Model: A Case Report. Iranian Rehabilitation Journal. 2021; } \\
\text { 19(1):111-116. http://dx.doi.org/10.32598/irj.19.1.1165.1 }\end{array}$ \\
\hline 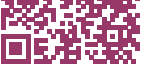 & doj'http://dx.doi.org/10.32598/irj.19.1.1165.1 \\
\hline
\end{tabular}

(c) (i) (8)

Article info:

Received: 30 Jun 2020

Accepted: 30 Mar 2021

Available Online: 01 Mar 2021

Keywords:

Laser therapy, Biomechanics, Gait, Diabetes, Case reports

\section{ABSTRACT}

A 49-year-old male patient with type 2 diabetes mellitus and Diabetic Foot Syndrome (DFS) was evaluated by comprehensive diabetic foot assessment. A 10 -session photobiomodulation therapy (low-level laser therapy) was prescribed for neuropathic pain and symptoms applying the scanning and probe method. Customized insoles were recommended for redistributing the plantar pressures and reducing stress on the diabetic foot's plantar surface for 4 weeks. Dynamic plantar pressure distribution, lower limb kinematics, and postural sway were evaluated using WinTrack dynamic pressure platform. Photobiomodulation therapy with customized insoles is useful in the redistribution and treatment of plantar pressure and gait kinematics. This approach should be extensively used in DFS as preventive and treatment measures.

* Corresponding Author:

G Arun Maiya, PhD.

Address: Centre for Diabetic Foot Care \& Research, Kasturba Hospital, MAHE (Deemed University \& Institute of Eminence), Manipal - 576104

Karnataka, India.

Tel: +98 (919) 845350823

E-mail: arun.maiya.g@gmail.co 


\section{Highlights}

- This study was the first attempt to incorporate photobiomodulation with the customized insole.

- Detailed biomechanical analysis was evaluated in this research.

- The explored clinical and biomechanical parameters indicated significant improvements.

\section{Plain Language Summary}

Individuals with type 2 diabetes mellites can develop symptoms, such as pain, burning sensation along increased pressure at their foot, which can cause ulcers. Numerous treatment approaches are were available for this condition. Noninvasive management, like laser treatment with adjusted insoles, can reduce pain and pressure in the feet.

\section{Introduction}

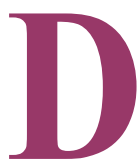

iabetic Foot Syndrome (DFS) is defined as a multi-factorial clinical triad of neurological, vascular, and musculoskeletal changes induced by diabetic peripheral neuropathy [1]. The overall prevalence of DFS, according to the International Working Group on the Diabetic Foot (IWGDF) Risk Classification System, is reported to be $51.8 \%$ in India; it is a common reason for hospitalizing patients with diabetes and its related complications [2,3]. Biomechanical changes and foot deformity due to motor neuropathy; the loss of protective sensation induced by sensory neuropathy; and autonomic alternations, such as fissures, callus, bunion contribute to the altered plantar pressure distribution and ankle kinematics of DFS. Accordingly, such conditions lead to an increase in the incidence of the generation of diabetic foot ulcers [4].

\section{Case Presentation}

A 49-year-old male patient with a 10-year history of type 2 diabetes mellitus presented to a diabetic foot clinic. His chief complaints were frequent burning and pricking sensation on feet for 3 years. He was under oral hypoglycemic pharmacotherapy for 5 years. His demographic findings are explained in Table 1. Besides, after providing an informed consent form, a comprehensive diabetic foot evaluation form was performed to obtain a detailed diabetic foot evaluation (Table 2). This study was approved by the Institutional Ethics Committee of Kasturba Medical College and Hospital (IEC169/2019).

At the baseline, dynamic plantar pressure distribution (3-sec duration, 100 images per second, $100 \mathrm{~Hz}$ ) and postural sway (30 sec 1200 images, $40 \mathrm{~Hz}$, one parameter) were evaluated using WinTrack dynamic pressure plate (WinTrack, France). The 3-step gait protocol indicated good reliability, with Intraclass Correlation Coefficient (ICC) values ranging between 0.75 and 0.90 in gait parameters. He was instructed to walk on the platform at a reasonable pace. Three trials were conducted, and the relevant data were extracted and analyzed by WinTrack software. Photobiomodulation therapy (low-level laser therapy) was prescribed for neuropathic pain and symptoms. A scanning laser wavelength of $632.8 \mathrm{~nm}$ with the dose of $3.1 \mathrm{~J} / \mathrm{cm}^{2}$ (TECH LASER SS-1000, Technomed, India) at the plantar and dorsal surface of the foot (6 min each), the probe laser wavelength of $660 \mathrm{~nm}$ and 850nm (THOR Laser Pro, THOR Photomedicine Ltd, USA) with the dosage of $3.4 \mathrm{~J} / \mathrm{cm}^{2}$ by contact method at popliteal fossa (Figure 1)*3 min were implemented. Ten regular sessions of photobiomodulation were conducted.

The LASER equipment was calibrated with a Laser Photometer Power meter kit. Customized insoles were recommended for redistributing the plantar pressures and reducing stress on the diabetic foot's plantar surface. The insole was developed by FootBalance equipment. FootBalance medical insoles were applied for molding. It is a $1.2 \mathrm{~mm}$ thick insole made with 4 layers. The outermost layer is a Durable top layer with an antibacterial coating. The middle layer is Comfortable, a high-quality shock absorption base layer. Initially, the study participant was requested to stand barefoot on the transparent mirror on the FootBalance platform and data analysis was conducted. Then, the study participant's foot arch was kept in 3 functional positions; standing straight, knees bent $45^{\circ}$ and standing with toes extension. Comparing their arch matching was performed by the closest type of medial arch type was selected. An appropriate foot size insole was selected and inserted into the heating rack. We placed the warm insole between the study par- 
Table 1. The demographic data of the study participant

\begin{tabular}{cc}
\hline Variables & Values \\
\hline Age, $\mathrm{y} /$ Gender & $49 / \mathrm{M}$ \\
\hline Weight, $\mathrm{kg}$ & 95.9 \\
\hline Height, $\mathrm{cm}$ & 186 \\
\hline BMI, $\mathrm{kg} / \mathrm{m}^{2}$ & 27.7 \\
\hline The duration of diabetes, $\mathrm{y}$ & 10.2 \\
\hline FBS & 127 \\
PPBS & 196 \\
\hline Blood pressure (systolic/diastolic), $\mathrm{mm} \mathrm{Hg}$ & $125 / 80$ \\
\hline
\end{tabular}

Iranian Rehabilitation \ournal

ticipant's foot and Fitpillow; subsequently, it was molded according to the arch and weight distributed evenly on both feet for 20 seconds. Then, a customized insole inside footwear was inserted (Figure 2).

The reassessment was performed at the end of the fourth week to compare the pre-post changes of vibra- tion perception threshold plantar pressure distribution changes and postural sway (Table 3 ).

\section{Discussion}

The present study data suggested a change in various biomechanical parameters. In the right foot, a reduction

Table 2. Clinical findings from a detailed diabetes foot evaluation

\begin{tabular}{ccc}
\hline On Observation & Right Foot & Left Foot \\
\hline Dry skin & Present & Present \\
\hline Altered skin color & Present & Present \\
\hline Ingrown nails & Present & Present \\
\hline Callus & Present & Absent \\
\hline Fissures & Present & Present \\
\hline On Examination & & Left Foot \\
\hline Skin temperature $\left({ }^{\circ} \mathrm{C}\right)$ & Right Foot & 32.8 \\
\hline Sensation & 33.1 & Present \\
\hline Ankle reflexes & Present & Diminished \\
\hline Pedal pulse & Diminished & Palpable \\
\hline Monofilament & Palpable & $4 / 6$ \\
\hline Vibration perception threshold $(\mathrm{V})$ & $3 / 6$ & 25 \\
\hline Ankle-brachial index & 32 & 1.05 \\
\hline
\end{tabular}




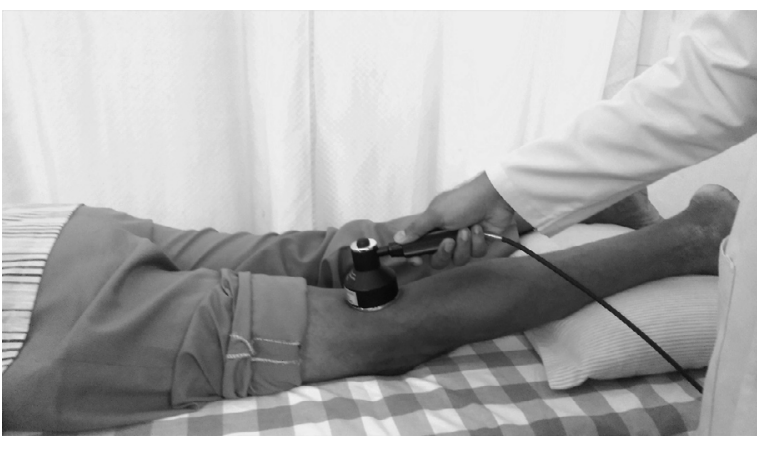

Iranian Rehabilitation Journa

Figure 1. The application of laser probe at the popliteal fossa

of APP $11.9 \%$, MPP to $6.5 \%$; and in the left foot, APP $11.1 \%$ and $31.2 \%$, respectively were detected. The total contact area of the right and left feet increased by $17.6 \%$ and $41.1 \%$, respectively. The forefoot hindfoot ratio is also a predictor of developing forefoot ulcers; the higher the ratio, the higher the risk. The combined intervention
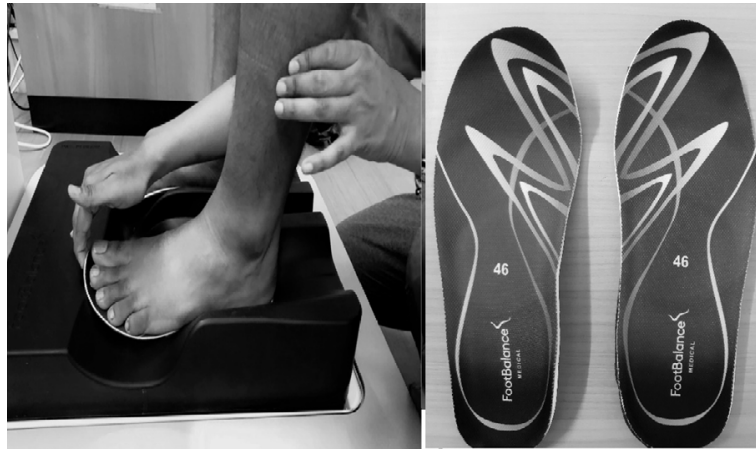

Figure 2. Customized insoles

Iranian Rehabilitation Journal

has reduced the ratio of foot pressures [5]. Vibration Perception Threshold (VPT) is a sensory test to evaluate the extent of the loss of protective sensation in diabetic peripheral neuropathy. Values more than $20 \mathrm{~V}$ were considered along with the inability to perceive $10 \mathrm{gr}$ monofilament for at least one point to be peripheral neuropathy. In

Table 3. Plantar pressure distribution parameters

\begin{tabular}{|c|c|c|c|c|}
\hline \multirow{2}{*}{ Variables } & \multicolumn{2}{|c|}{ Right Foot } & \multicolumn{2}{|c|}{ Left Foot } \\
\hline & Pre-intervention & Post-intervention & Pre-intervention & Post-intervention \\
\hline Average plantar pressure ( $\mathrm{kPa})$ & 126.8 & 111.7 & 120.1 & 106.7 \\
\hline Maximum Plantar Pressure (kPa) & 293.6 & 274.4 & 316.9 & 217.7 \\
\hline Pressure. Time Integral (kPa.s) & 67.52 & 51.33 & 57.07 & 36.36 \\
\hline F/H Ratio & 1.40 & 1.45 & 1.22 & 1.41 \\
\hline Step duration (ms) & 720 & 755 & 510 & 655 \\
\hline Swing duration(ms) & 1360 & 1565 & 1360 & 1615 \\
\hline Step length (mm) & 578 & 578 & 422 & 508 \\
\hline Gait cycle duration (ms) & 1000 & 1410 & 1000 & 1412 \\
\hline Total contact area $\left(\mathrm{cm}^{2}\right)$ & 102 & 127 & 99 & 140 \\
\hline Stride duration (ms) & \multicolumn{2}{|c|}{2080} & \multicolumn{2}{|c|}{2370} \\
\hline Gait cycle length (mm) & \multicolumn{2}{|c|}{1230} & \multicolumn{2}{|c|}{1086} \\
\hline Double stance duration (ms) & \multicolumn{2}{|c|}{200} & \multicolumn{2}{|c|}{305} \\
\hline CoP length (mm) & \multicolumn{2}{|c|}{323.4} & \multicolumn{2}{|c|}{273.3} \\
\hline CoP Area (mm) & \multicolumn{2}{|c|}{365.2} & \multicolumn{2}{|c|}{198.7} \\
\hline Length/Area (per mm) & \multicolumn{2}{|c|}{0.9} & \multicolumn{2}{|c|}{1.4} \\
\hline$X$ speed $(\mathrm{mm} / \mathrm{s})$ & \multicolumn{2}{|c|}{7.5} & \multicolumn{2}{|c|}{6.1} \\
\hline $\mathrm{Y}$ speed $(\mathrm{mm} / \mathrm{s})$ & \multicolumn{2}{|c|}{6.2} & \multicolumn{2}{|c|}{5.5} \\
\hline
\end{tabular}


the first 10 sessions, VPT has been improved from $32 \mathrm{~V}$ in the right to $25 \mathrm{~V}$ in the left to $9 \mathrm{~V}$ and $7 \mathrm{~V}$, respectively.

Kinematic parameters were also improved from pre- to post-intervention; especially stride duration, gait cycle length, and double stance duration. This finding reflects that the gait pattern was improved. Furthermore, the gait alterations were reduced for better propulsion of the foot during gait [6]. Balance is also the main component altered in diabetic peripheral neuropathy. Postural sway is among the gold standard methods with quantifiable variables. Variables, such as CoP length, CoP Area, X, and Y-axis speed, Y-axis deviation, have significantly improved in the explored patient. CoP length revealed increased postural stability in the patient. This may be attributed to enhanced plantar sensory perception induced by elevated neural activity at the lower peripheral nerves.

In this study, we have explored static and kinetic spatiotemporal parameters. Moreover, kinetic and kinematic characteristics were examined by the biomechanical model generated in the gait pattern. Photobiomodulation therapy is a noninvasive non-pharmacological modality based on the principle of bio-stimulation effect. Thus, PBMT significantly improves sensory perception. The potential mechanism might be due to PBMT that may prevent motor cell degeneration; induce Schwann cell proliferation; boost neural metabolism, and increase myelination and axon regeneration $[7,8]$.

For individuals with diabetic neuropathy, custom insoles are frequently prescribed to offload high pressure from the Metatarsal Heads (MTHs) and other regions that reduce the risk of plantar ulceration [9]. Insoles provide the critical interface between the foot and the shoe; they also present the most effective approach to minimizing potentially damaging tissue tension on the plantar part of the foot along with outsole modification [10]. The Diabetic Feet Australia guidelines on footwear for individuals with diabetes also recommended the appropriate footwear with customized insoles can reduce the plantar pressures at the plantar surface of diabetic foot. It also highlighted the reduction of $>30 \%$ in the region with the highest plantar pressure, compared to the same region of foot considered as plantar pressure $d$ effect [11].

\section{Conclusion}

Photobiomodulation therapy with customized insoles is useful in the redistribution and treatment of plantar pressure and gait kinematics. It should be extensively employed in DFS as preventive and treatment measures. However, this study was a case report, further detailed analysis and experimental study designs with long-term follow-up would help to strengthen this argument regarding the effectiveness of this approach.

\section{Ethical Considerations}

Compliance with ethical guidelines

There were no ethical considerations to be considered in this research.

\section{Funding}

This research did not receive any grant from funding agencies in the public, commercial, or non-profit sectors.

\section{Authors' contributions}

All authors equally contributed to conducting the study and drafting the manuscript.

\section{Conflict of interest}

The authors declared no conflicts of interest.

\section{Acknowledgments}

The authors would like to acknowledge the Centre for Diabetic Foot Care and Research (CDFCR), Manipal Academy of Higher Education (MAHE), Manipal, Karnataka, India.

\section{References}

[1] Volmer-Thole M, Lobmann R. Neuropathy and diabetic foot syndrome. International Journal of Molecular Sciences. 2016; 17(6):917. [DOI:10.3390/ijms17060917] [PMID] [PMCID]

[2] Vibha SP, Kulkarni MM, Ballala AK, Kamath A, Maiya GA. Community-based study to assess the prevalence of diabetic foot syndrome and associated risk factors among people with diabetes mellitus. BMC Endocrine Disorders. 2018; 18:43. [DOI:10.1186/s12902-018-0270-2] [PMID] [PMCID]

[3] Korada H, Maiya A, RaoSK, Hande M. Effectiveness of customized insoles on maximum plantar pressure in diabetic foot syndrome: A systematic review. Diabetes \& Metabolic Syndrome 2020; 14(5):1093-9. [DOI:10.1016/j.dsx.2020.06.041] [PMID]

[4] Armstrong DG, Boulton AJ, Bus SA. Diabetic foot ulcers and their recurrence. New England Journal of Medicine. 2017; 376(24):2367-75. [DOI:10.1056/NEJMra1615439] [PMID]

[5] Caselli A, Pham H, Giurini JM, Armstrong DG, Veves A. The forefoot-to-rearfoot plantar pressure ratio is increased in severe diabetic neuropathy and can predict foot ulcera- 
tion. Diabetes Care. 2002; 25(6):1066-71. [DOI:10.2337/diacare.25.6.1066] [PMID]

[6] Raspovic A. Gait characteristics of people with diabetesrelated peripheral neuropathy, with and without a history of ulceration. Gait \& Posture. 2013; 38(4):723-8. [DOI:10.1016/j. gaitpost.2013.03.009] [PMID]

[7] Bashiri H. Evaluation of low level laser therapy in reducing diabetic polyneuropathy related pain and sensorimotor disorders. Acta Medica Iranica. 2013; 51(8):543-7. [PMID]

[8] Fallah A, Mirzaei A, Gutknecht N, Demneh AS. Clinical effectiveness of low-level laser treatment on peripheral somatosensory neuropathy. Lasers in Medical Science. 2017; 32(3):721-8. [DOI:10.1007/s10103-016-2137-y] [PMID]

[9] Lewis J, Lipp A. Pressure-relieving interventions for treating diabetic foot ulcers. Cochrane Database of Systematic Reviews. 2013; (1):CD002302. [DOI:10.1002/14651858. CD002302.pub2] [PMID]

[10] Boulton AJM. Therapeutic footwear in diabetes. Current Diabetes Reports. 2002; 2:475-6. [DOI:10.1007/s11892-002-0115-1]

[11] Van Netten JJ, Lazzarini PA, Armstrong DG, Bus SA, Fitridge R, Harding K, et al. Diabetic foot Australia guideline on footwear for people with diabetes. Journal of Foot and Ankle Research. 2018; 11:2. [DOI:10.1186/s13047-017-0244-z] [PMID] [PMCID] 